\title{
Control Issues of Digital Clay - Massive Hydraulic Actuator Array for Man-machine Communication
}

\author{
Haihong Zhu \\ School of Mechanical Engineering \\ Georgia Institute of Technology \\ Atlanta GA 30332-0405 USA \\ Haihong.zhu@me.gatech.edu
}

\author{
James D Huggins \\ School of Mechanical Engineering \\ Georgia Institute of Technology \\ Atlanta GA 30332-0405 USA \\ James.Huggins@mail.gatech.edu
}

\author{
Wayne J. Book \\ School of Mechanical Engineering \\ Georgia Institute of Technology \\ Atlanta GA 30332-0405 USA \\ wayne.book@me.gatech.edu
}

\begin{abstract}
Digital Clay is an NSF funded project for human machine communication through a tangible haptic surface

fluidic driving system, and control system. Focus of this paper is the control of some of the special mechanisms of Digital Clay. Specifically, the Fluid Matrix Drive, Capacitive-Coupled Resistive Sensing technology for position feedback and the other control related issues are discussed. Analysis and testing results on these topics are provided based on a $5 \times 5$ actuator array prototype. Most of the topics discussed in this paper are widely applicable to other devices as well as Digital Clay.
\end{abstract}

Keywords: Haptics, Human-machine interface, Massive Actuator Array, Control

\section{INTRODUCTION}

\section{Digital Clay Concept}

Digital Clay is proposed as a human-machine interface to combine the advantages of both digital computation on the computer side and physical models on the human side (e.g. modeling clay). Digital Clay provides a 3D tangible haptic surface formed by a massive array of cells (miniature hydraulic actuators with sensors). This surface can be maneuvered bilaterally by the computer and/or by the hands of the user as depicted in Fig 1.

Sophisticated forms of digital representation and analysis offer great value during research and design. But they are more suitable for the later phases of design where greater precision and quantitative analysis are required. During earlier design phases, there is value in the use of physical models in the planning and design process that can be lost if models only have numerical representation. This is simply because a physical shape is more intuitive for humans helping understand complex geometries and physical relationships.

To bridge the gap between the computer-aided design and the physical tangible modeling, Digital Clay provides a novel human-computer interface based on a tangible haptic $2.5 \mathrm{D}$ and reformable surface that can be shaped by the user and immediately acquired by a computer or shaped by the computer for the user to examine. In this sense, Digital Clay can be deemed as a bilateral man-machine communication device, through the means of displacement and force. actuated using fluid power. Digital Clay's hardware can be divided into three subsystems: massive-integrated actuator-sensor array,

Digital Clay's hardware is divided into three subsystems: 1) a massive-integrated fluid actuator-sensor array, 2) a fluidic driving system and 3) an electronic controller. Digital Clay's control is organized into three levels: 1) application programming interface (User API), 2) surface control, which considers cell to cell interaction and coordinates the cell level controls, and 3) cell level, which commands individual valves based on the received commands and sensor feedback.

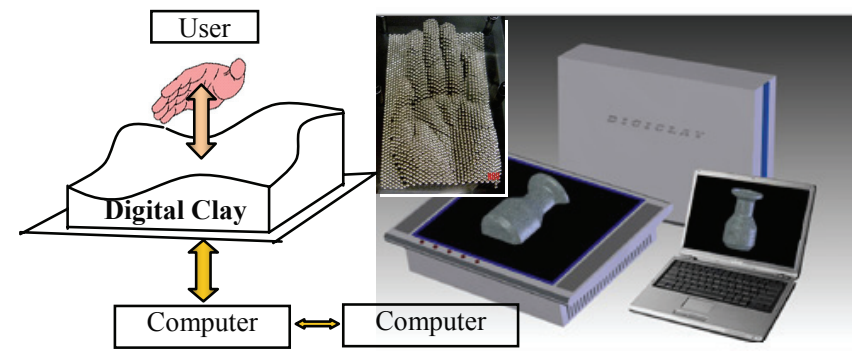

Fig 1 Concept of Digital Clay

The potential applications of Digital Clay cover a wide range including $\mathrm{CAD}, 3 \mathrm{D}$ mapping, medical diagnostics, scientific research, assistance for the visually impaired and entertainment.

\section{Background and Context}

Attempts and approaches most relevant to Digital Clay are reviewed and presented here. A tactile array is a device typically constructed by a planar arrangement of linear actuators and sensors acting perpendicularly to that plane. In some aspects, Digital Clay is similar to tactile sensors with tactile stimulators [1] [2] except Digital Clay has a longer stroke and operates hydraulically. Electromagnetic [3], pneumatic [4] and shape memory alloy [5] actuators have been used in tactile arrays. The sizes of these arrays are small, about the size of the pad of a finger. The device by Kammermeier [3], for example, covers $16 \times 16 \mathrm{~mm}$ with 36 cells. However, the displacement of these tactile devices is too small for a shape display. Project POPUP! [6] sought to display $3 \mathrm{D}$ objects by orchestrating the vertical motion of a dense array of pin-rods actuated using Shape Memory Alloy (SMA) actuators. SMA essentially works through a temperature change. Heat transfer is not as prompt as electricity and fluid power. Therefore, the response is relatively slow. Furthermore, force control is 
difficult. Project FEELEX [7] (Fig 2) was reported by Hiroo Iwata in 1998. The prototype comprises an array of pin-rods actuated by electrical motors. The main problem for any device using electrical actuators is the force and displacement the actuator provides. Since motors rotate at high speeds for efficient operation, a speed reduction mechanism is needed and, thus, leads to low force density and low resolution across the plane.

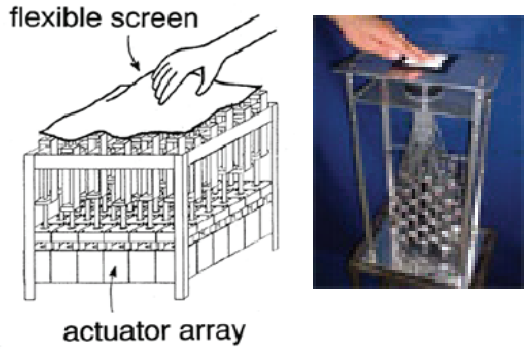

Fig 2 Project FEELEX

\section{Control Challenges of Large Numbers of Units}

Digital Clay is a system with controlled units distributed over a spatial domain that operate asynchronously. Related examples can be found in Intelligent Vehicle Highway Systems and Air Traffic Management Systems [8]. However, with the potential scale of thousands or millions of cells, centralized control becomes unviable due to the challenges of gathering information, centrally computing the results and distributing the commands for action. Decentralized control, on the other hand, using only local information while guaranteeing stability of the entire system is more suitable for this kind of large scale system. There are three types of decentralized control structures: fully-, partially- and hierarchically decentralized control [9] [10] [11]. The methods chosen for controlling Digital Clay come from a modified version of decentralized control and from large scale LED matrix drive concepts. An LED array Matrix Drive controls each LED using a row and column addressing scheme. However, since the hydraulic actuator used here is different from an electrical component, this method is modified before application.

This paper describes the control issues relevant to the essential components of Digital Clay. The novel fluidic driving system (Fluidic Matrix Drive) and its control (i.e. basic surface control) are first discussed and followed by a discussion of the massive-integrated hydraulic actuator array. Since the sensors are embedded with each actuator, the sensor system will be introduced at the same time as the actuator array. The high level control is more conventional compared to the actuator array, sensing and the driving system. Therefore, the high level control will be discussed only briefly.

Digital Clay is a large project sponsor by the NSF. Being limited by space, this paper focuses on the essential and exclusive control issues mentioned above. More details about the hardware and low level control can be found in conference publications [12-14] and a $\mathrm{PhD}$ thesis [15].

\section{FLUIDIC MATRIX DRIVE AND CONTROL}

\section{Concept of Fluidic Matrix Drive (FMD)}

To drive a massive-integrated actuator array, conventional fluid driving scheme face overwhelming problems including cost and control resource limitations. In this section, the Fluidic Matrix Drive is discussed and followed by two basic control methods. Another prospective control algorithm is introduced in the future work section of this paper. (FMD is a patent pending applied for by Georgia Tech.)

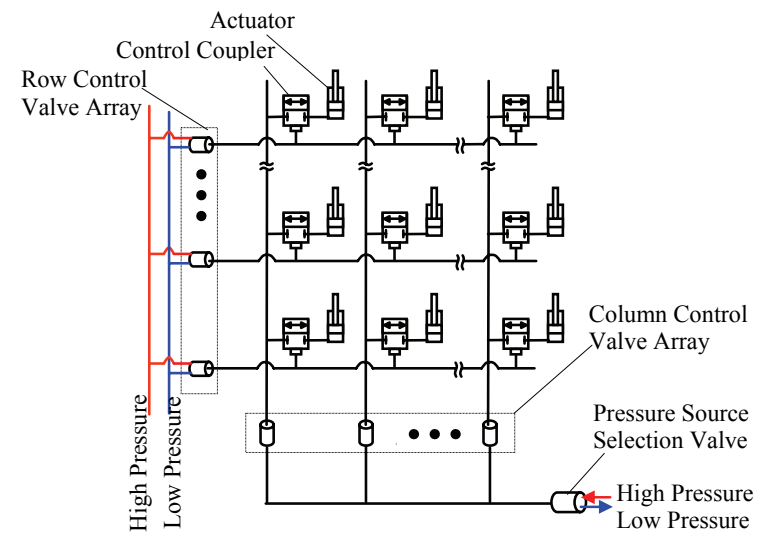

Fig 3 Hydraulic Matrix Drive Scheme

To achieve a large, high-resolution display, a tremendous number of cells are required. (The term "cell" is used to represent the actuator and its sensors/electronics, etc.) As a result, manufacturing and control of the device will be unviable through conventional approaches. To solve the problem, a scheme called the Fluidic Matrix Drive (FMD) is proposed and implemented. FMD is composed of two 1-D arrays of control valves and a 2-D array of control couplers. As shown in Fig 3, the row control valves and the single pressure source selection valve are 2-position/3-way solenoid valves. The common ports of these valves are connected to the array of actuators and the other two ports are connected to the high pressure source and the drain. The control coupler is essentially a 2-position/2-way fluid driven valve. Since the control coupler is much easier and cheaper to fabricate than solenoid valves, the control coupler is not considered as a control valve but as a coupler.

Using the FMD approach, every actuator in an $\mathrm{M} \times \mathrm{N}$ actuator array can be independently driven using only $(\mathrm{M}+\mathrm{N}+1)$ valves instead of $2 \times \mathrm{N} \times \mathrm{M} 3$-way valves or $\mathrm{N} \times \mathrm{M}$ 4-way valves. For instance, a $100 \times 100$ cell array needs only 201 valves instead of 20,000 3-way valves, or 10,000 4-way valves.

\section{Working Principle}

As shown in Fig 3, each row control valve controls the on-off of the control couplers in one row. Each control coupler controls the passage from the column control valve to the corresponding actuator. As a consequence, a specific actuator can be activated only when the corresponding row valve and its corresponding column valve are both enabled. The process is illustrated in the Fig 4. By its nature, FMD drives the whole surface in a manner of refreshing the actuators in one row and followed by another row just like 
that refreshing a monitor or TV. When a certain row valve is enabled, all the actuators on that row are then controlled by their corresponding column valves. Pulse Width Modulation is applied on each column valve to achieve different moving speeds of each actuator.

Though similar to an LED matrix drive, FMD has limitations. One is the time delay when switching between row valves because electricity conducts much faster than the fluid media used by the FMD. Furthermore, LEDs respond to the electrical signal much faster than the control coupler. To identify limitations on refresh speed, preliminary experiments were conducted. The experimental results show that: even with a gap $d_{1}$ as small as $0.5 \mathrm{~mm}$ and a channel diameter $d_{2}$ of $1 \mathrm{~mm}$, the velocity of the actuator is still more than $100 \mathrm{~mm} / \mathrm{sec}$. This is simply because the bore size of the actuator is only $3 \mathrm{~mm}$ in diameter. Therefore, basic theoretical analysis is conducted only on the row control chain.

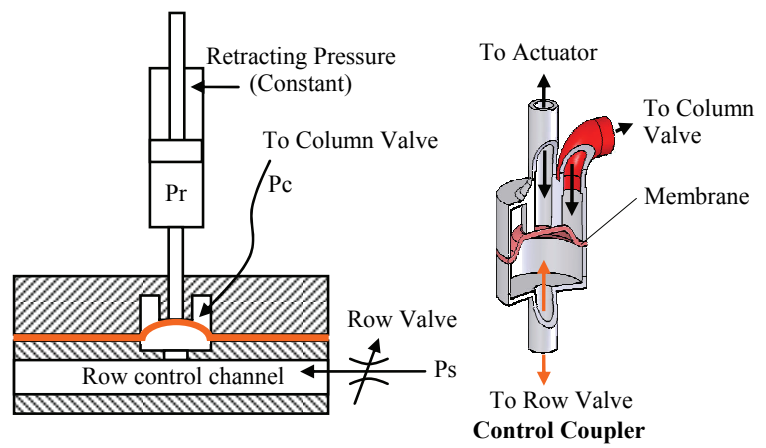

a) Disabled

Control Coupler

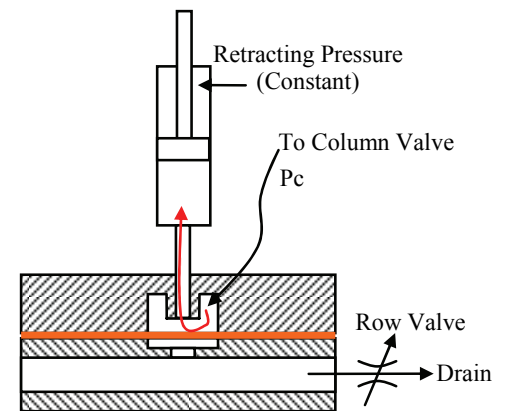

b) Enabled

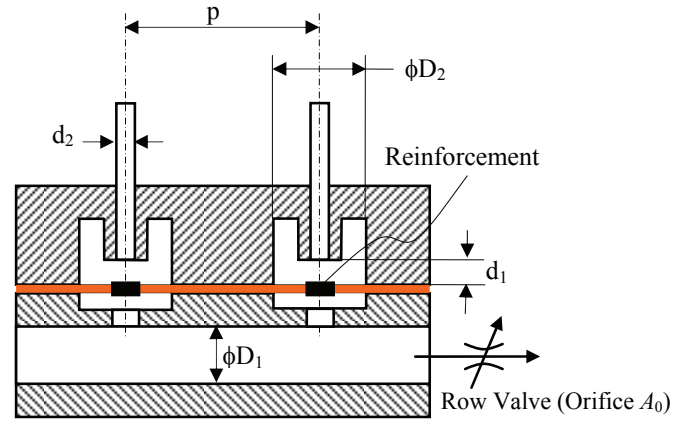

c) Dimensions

Fig 4 Working Principle of the Control Adapter

As shown in Fig $4 \mathrm{a}$ and $4 \mathrm{~b}$, the time for a row to be refreshed includes: 1) time $t_{1}$ to enable all the control couplers, 2) time $t_{2}$ for all actuators in that row to reach reference positions, and 3 ) time $t_{3}$ disable all the control couplers. As mentioned, $t_{2}$ is less affected by the structure of FMD. Therefore, only $t_{1}$ and $t_{3}$ are investigated.
The fluid volume change between control coupler enable and disable states is caused by the bumping out of the diaphragm. From solid mechanical analysis the volume change can be calculated as below. The reinforcement on the diaphragm prevents the diaphragm from getting into the holes. In addition, the pocket under the diaphragm is quite small and is shown larger here just for illustration.

$$
\begin{aligned}
& \quad \Delta V=n \cdot \Delta v=2 n \pi d_{1} \int_{0}^{D_{2} / 2}\left(1-\frac{r^{2}}{\left(D_{2} / 2\right)^{2}}\right)^{2} r d r \\
& \quad \Rightarrow \Delta V=\frac{n \pi d_{1} D_{2}^{2}}{12}=n \cdot 2.094\left(\mathrm{~mm}^{3}\right)=\mathrm{n} \cdot 2.094 \times 10^{-3}(\mathrm{~mL}) \\
& n=\text { the number of control couplers in that row; } \\
& d_{1}=0.5 \mathrm{~mm}, D_{2}=4 \mathrm{~mm}, \text { according to the actual system }
\end{aligned}
$$

In reality, the row control channel cross section is much larger than the orifice $\left(A_{0}\right)$ of the row control valve $\left(6 \mathrm{~mm}^{2}\right.$ v.s. $0.36 \mathrm{~mm}^{2}$ in the current design). Therefore, the flow rate is mainly subjected to the valve orifice. To get a more accurate flow estimation, the calculation method suggested by the valve manufacturer (The LEE Company) is used here. During the enable process, the flow rate (using water as the media since it is clean for human users) is calculated below. Note, before draining the fluid in the row control channel, the column valves are opened to get an actuation pressure $P_{c}$ higher than the retracting pressure $P_{r}$. (Fig 4a)

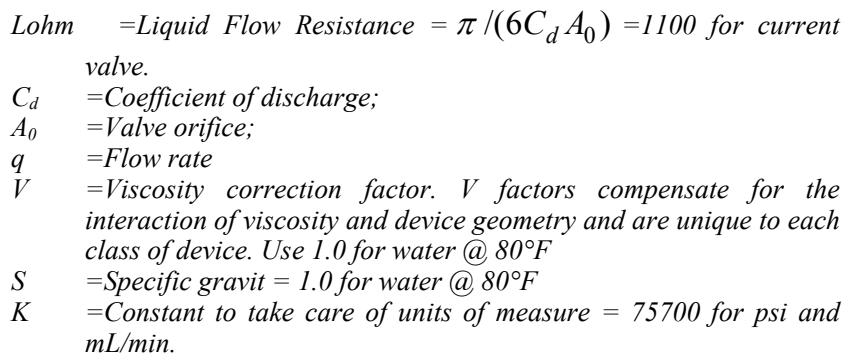

Using actual values of the current system, i.e., $n=5, P_{c}=$ 20 PSI, the time during enable period is:

$$
t_{1}=\Delta V / q=0.4 m s
$$

During the disable period, all the column valves are closed first. The pressure difference across the row valve orifice is $\left(P_{s}-P_{r}\right)$. In the real system, $P_{s}=30$ PSI, $P_{r}=10$ PSI, $t_{3}$ can be found using the above approach as $0.4 \mathrm{~ms}$. More details about the flow rate calculation and the definition of parameters can be found at the valve manufacturer's website: www.theleeco.com. The model of the control valve is LHDA1223111H.

Besides water, working fluids such as silicon oil and air are also considered. Because of the compressibility of air, the disable period has two parts: charging the dead volume (i.e. the row control channel, etc.) and inflating the diaphragm after the pressure reaches $P_{r}$. To reduce the charging time for the dead volume, the drain pressure is not atmosphere, but a pressure $P_{r}$ ' slightly lower than $P_{r}$. An analysis is shown below.

\section{Enable period}

$$
\begin{aligned}
& \text { Upstream absolute pressure } P_{1}=P_{c}+P_{\text {atm }}=34.7 \text { PSIA } \\
& \text { Downstream absolute pressure } P_{2}=P_{r}{ }^{\prime}+P_{\text {atm }}=22.7 \text { PSIA } \\
& P_{1} / P_{2}<1.9, \Rightarrow \text { Sub }- \text { Sonic Flow }
\end{aligned}
$$




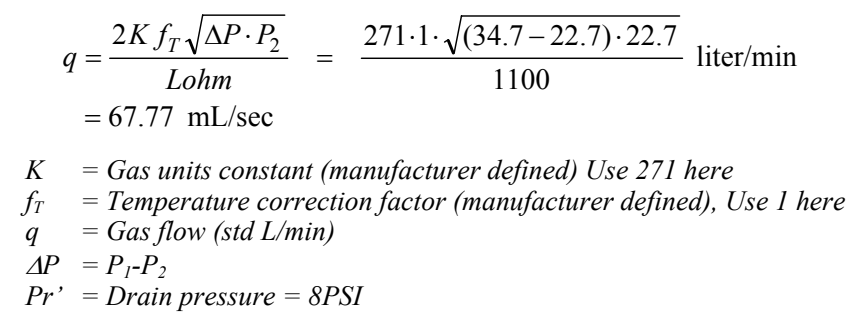

Therefore,

$$
t_{1}=\Delta V / q=0.15 \mathrm{~ms}
$$

\section{Disable period}

As previously mentioned, since the downstream pressure $P_{r}{ }^{\prime}$ is close to the $P_{r}$, the time for charging the dead volume is negligible.

$$
\begin{aligned}
q & =\frac{2 K f_{T} \sqrt{\Delta P \cdot P_{2}}}{L o h m}=\frac{271 \cdot 1 \cdot \sqrt{(44.7-24.7) \cdot 24.7}}{1100} \text { liter } / \mathrm{min} \\
& =91.26 \mathrm{~mL} / \mathrm{sec}
\end{aligned}
$$

Therefore,

$$
t_{3}=\Delta V / q=0.11 \mathrm{~ms}
$$

In the above analysis, the length of the row control channel is not considered because the total length is short (less than 1 meter). The deformation of the diaphragm may vary, but will not be dramatically different from the ideal deformation calculated above. Note that the above analysis is based on the $5 \times 5$ cell array. For a $100 \times 100$ cell array, the time is 20 times longer, i.e., $20 \cdot\left(t_{1}+t_{3}\right)=5.2 \mathrm{~ms}$ for air and $16 \mathrm{~ms}$ for water for one row to enable and disable. However, consider the cost reduction from 20,000 valves to 201 valves, there is a trade-off between the costs and refresh speed. The analysis also indicates that the bottle neck of the control coupler actuation speed is the on-off time of the row control valve. The current row control valve has an on/off time of $5 \sim 10 \mathrm{~ms}$, which is slower than the control coupler response.

Choosing liquid or air as the fluid media depends on the application. When using air, if the external force on the actuator is large enough, the diaphragm will deflect causing the fluid in the actuator to flow into other actuators on the same column. This may not be preferred for some applications, but may help for Digital Clay, which will be introduced in the future work section.

\section{Realization of FMD}

A practical realization of the FMD for massive actuator array is shown in Fig 5. The hydraulic driving manifold is composed of two blocks: an upper block and a lower block. Chambers built in these blocks along with a membrane between them form the control coupler array (Fig 4a). On the $5 \times 5$ prototype (Fig 10 ), the FMD is realized by two parts fabricated using stereo lithography technology and a silicon rubber sheet (for diaphragm).

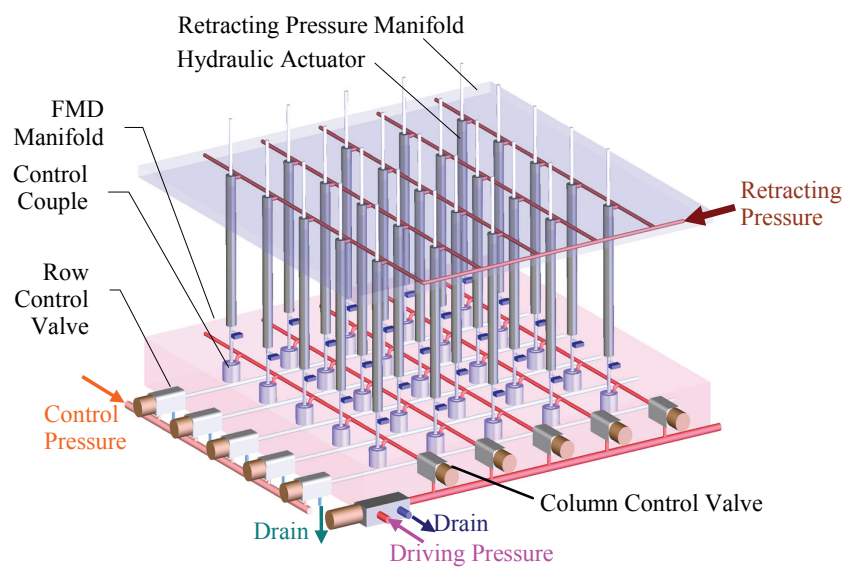

Fig 5 FMD for Massive-integrated Actuator Array

\section{Surface Refresh Methods}

An operation $\Theta$ is defined under following rule:

$$
\begin{aligned}
& \mathrm{A}=\left[\begin{array}{c}
a_{1} \\
\vdots \\
a_{i} \\
\vdots \\
a_{n}
\end{array}\right] ; \text { and } \mathrm{B}=\left[\begin{array}{lllll}
b_{1} & \cdots & b_{j} & \cdots & b_{n}
\end{array}\right] ; \\
& \text { Then } \mathrm{A} \Theta \mathrm{B}=\left[\begin{array}{ccc}
\mathrm{g}\left(a_{1}, b_{1}\right) & \mathrm{g}\left(a_{1}, b_{2}\right) & \cdots \\
\mathrm{g}\left(a_{2}, b_{1}\right) & \ddots & \\
\vdots & \ddots & \mathrm{g}\left(a_{i}, b_{j}\right)
\end{array}\right] \\
& \text { where, } a_{i}=f_{1}\left(\delta_{i}\right) ; b_{j}=f_{2}\left(\delta_{j}\right) ; 0 \leq \mathrm{a}_{\mathrm{i}}, \mathrm{b}_{\mathrm{j}} \leq 1
\end{aligned}
$$

Where, $\delta_{i}$ and $\delta_{j}$ are the PWM duty cycles on row and column valves respectively. Functions $f_{z}\left(\delta_{k}\right)(z=1,2)$ depends on variables such as pressure drop, discharge coefficient, etc. Function $\mathrm{g}(\mathrm{x}, \mathrm{y})$ produces the actuator's speed as a result of the row and column PWM duty cycles. At the current stage, only full open (i.e., $\delta_{i}=0, \mathrm{a}_{\mathrm{i}}=0$ ) and/or full close (i.e., $\delta_{i}=100 \%, \mathrm{a}_{\mathrm{i}}=1$ ) for row control valves are considered. Different PWM duty cycles are applied to the column valves to control the speed of each actuator in an enabled row. In other words, if $x=1, g(x, y)$ $=\mathrm{y}$ and if $\mathrm{x}=0, \mathrm{~g}(\mathrm{x}, \mathrm{y})=0$.

Before describing detailed refresh methods, one issue that needs clarification is that refreshing methods only provide the reference inputs. There are mechanisms embedded in the on-board controller to perform PD control for each cell during any specific row refresh process. Once a row is enabled, all the cells on that row may reach their reference position simultaneously using Pulse Width Modulation on the column valves.

\section{One-time Refresh Method}

Using the one-time refresh method, actuators are controlled to reach their final positions row by row. For example, let the desired final surface matrix, $\mathrm{C}$, be: 


$$
\mathrm{C}=\left[\begin{array}{lllll}
0 & 1 & 2 & 3 & 4 \\
0 & 0 & 1 & 2 & 3 \\
0 & 0 & 0 & 1 & 2 \\
0 & 0 & 0 & 0 & 1 \\
0 & 0 & 0 & 0 & 0
\end{array}\right]
$$

Assume the maximum speed of current actuator is 5 units/sec; refresh settling time (for control couplers to open and close) is $0.02 \mathrm{sec}$. No action is needed for the first row (numbered from bottom to top). The vectors for controlling the valve arrays for the $2^{\text {nd }}$ row refresh are $\mathrm{A} 1$ and $\mathrm{B} 1$, and the surface matrix is $\mathrm{C} 1$.

$$
\begin{gathered}
\mathrm{A} 1=\left[\begin{array}{lllll}
0 & 0 & 0 & 1 & 0
\end{array}\right]^{\prime} ; \quad \mathrm{B} 1=\left[\begin{array}{lllll}
0 & 0 & 0 & 0 & 1
\end{array}\right] ; \\
\mathrm{C} 1=\mathrm{A} 1 \Theta \mathrm{B} 1 * t_{1}=\left[\begin{array}{ccccc}
0 & 0 & 0 & 0 & 0 \\
0 & 0 & 0 & 0 & 0 \\
0 & 0 & 0 & 0 & 0 \\
0 & 0 & 0 & 0 & 1 \\
0 & 0 & 0 & 0 & 0
\end{array}\right] ; \quad \text { where } t_{1}=0.2 \mathrm{sec}
\end{gathered}
$$

Similarly, for 3rd, 4 th row and 5th row:

$$
\begin{aligned}
& \mathrm{A} 2=\left[\begin{array}{lllll}
0 & 0 & 1 & 0 & 0
\end{array}\right]^{\prime} ; \quad \mathrm{B} 2=\left[\begin{array}{ccccc}
0 & 0 & 0 & 0.5 & 1
\end{array}\right] ; \\
& \mathrm{C} 2=C 1+\mathrm{A} 2 \Theta \mathrm{B} 2 * t_{2} \quad \text { where } t_{2}=0.4 \mathrm{sec} \\
& \mathrm{A} 3=\left[\begin{array}{lllll}
0 & 1 & 0 & 0 & 0
\end{array}\right] ; \quad \mathrm{B} 3=\left[\begin{array}{lllll}
0 & 0 & 1 / 3 & 2 / 3 & 1
\end{array}\right] \text {; } \\
& \mathrm{C} 3=C 2+A 3 \Theta B 3 * t_{3}, \quad \text { where } t_{3}=0.6 \mathrm{sec} \\
& \mathrm{A} 4=\left[\begin{array}{lllll}
1 & 0 & 0 & 0 & 0
\end{array}\right] ; \quad \mathrm{B} 4=\left[\begin{array}{lllll}
0 & 0.25 & 0.5 & 0.75 & 1
\end{array}\right] ; \\
& \mathrm{C} 4=C 3+A 4 \Theta B 4 * t_{4}, \quad \text { where } t_{4}=0.8 \mathrm{sec}
\end{aligned}
$$

The one-time refresh method is the simplest surface refresh method. It shows an obviously discontinuous generation of the surface.

\section{Gradual Refresh Method}

Cumulatively generating the final surface through several intermediate surfaces can provide a better and smoother transient performance. The easiest way to realize this is to divide the final surface matrix equally into intermediate matrices. However, this will increase the total time to achieve the final surface. Using a gradual refresh method, the total number of on/off state changes of the controller couplers increases with the number of intermediate surface matrices.

One way to reduce the number of control coupler state changes is to use the technology similar to interlacing for TV or monitor. Each time only half (or less) of the rows are refreshed. During each intermediate surface refresh, rows to be refreshed and rows not to be refreshed are mixed together to give a relatively smooth intermediate surface. Unlike conventional interlacing technology for monitor or $\mathrm{TV}$, curvatures of the final surface are also used to determine which actuators are to be refreshed. For instance, to generate a mountain shape, actuators near the peak will refreshed more times than the actuators near the root.

Generally speaking, to determine the intermediate surface matrix, trade offs between following criteria need to be made: 1) the affordable total refresh time to reach the final surface, and 2) the maximum displacement discrepancy that can be tolerated between adjacent pixels (i.e., the actuator tip). Please note that this may not be a fixed value. It also depends on the curvature of the final surface. For instance, human users may not pay attention rugged areas (big curvature) compared to those smooth areas, because they expect the roughness.

Quantitative analysis on the generation of intermediate surface is still ongoing. On the $5 \times 5$ prototype presented in this paper, only the one time refresh method is used.

\section{ACTUATOR-SENSOR ARRAY}

\section{Actuator Array}

The actuator array is formed by a planar $x-y$ array of linear actuators acting in the $\mathrm{z}$ direction as shown in Fig 6 . A miniature linear cylinder is designed. The actuator is composed of a glass (can be replaced by composite) tube (4mm OD and $3.2 \mathrm{~mm} \mathrm{ID)} \mathrm{and} \mathrm{a} \mathrm{graphite} \mathrm{piston.} \mathrm{Retraction}$ of the actuator is realized by a constant retracting pressure, as shown in Fig 6.

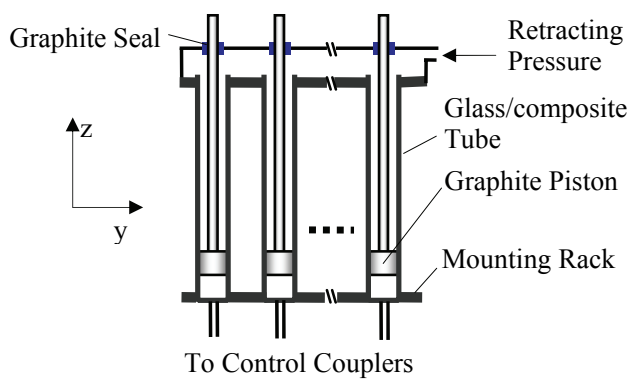

Fig 6 Structure of the Actuator Array

\section{Capacitive-coupled Resistive Sensing (CCRS)}

No suitable sensor for the array size and resolution was found, prompting the efforts to design a new displacement sensor. No off the shelf position sensor meets the size requirement of OD less than $1 \mathrm{~mm}$ and stroke longer than 50 mm. After evaluation of a number of concepts, the capacitive-coupled resistive sensing technology was conceived and implemented (Fig 7). It behaves like a noncontacting potentiometer. However, unlike a potentiometer, a CCRS sensor uses the capacitance between the resistive member and the sensing head instead of the physical contacting wiper in a potentiometer.

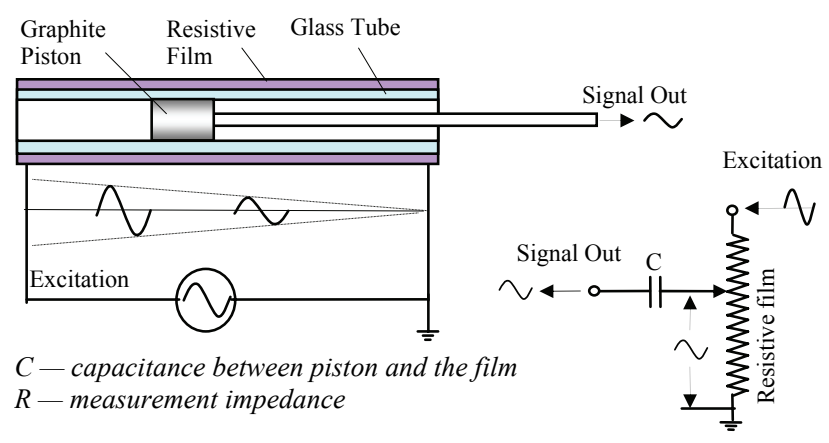

Fig 7 Capacitive-coupled Position Transducer

As shown in Fig 7, a uniform resistive film is deposited outside the glass tube. An AC voltage is applied across the resistive film. Obviously, the amplitude of AC voltage along the cylinder is linearly distributed. The AC voltage is then coupled by the capacitance between the film and the 
graphite piston. By measuring the amplitude of $\mathrm{AC}$ voltage on the piston, its position can be detected. The actual sensor also includes shielding and conducting mechanisms to conduct the signal from the piston to the signal conditioner and, therefore, is slightly complicated. Stand alone CCRS sensors have the same or higher performance (i.e., repeatability, anti-wear and linearity) compared to LVDT but cost less than linear potentiometer sensors $(\sim \$ 15$ not including signal conditioner). Because of its merits, the CCRS technology is patented by Georgia Tech and being commercialized by Sentrinsic LLC. It is currently being embedded into products of commercial companies.

Since the output voltage is alternating, a signal conditioner is required to convert the alternating voltage into a DC voltage representing the piston position along the resistive member. In reality, the position sensor is excited by a square wave instead of sinusoidal waveform shown in Fig 7. The advantages of using a square wave include easy generating of the excitation voltage, easy demodulating of the output signal, and higher accuracy.

The sensor itself is very simple to embed into the actuator array. Recent experiments successfully embedded 25 sensors with effort and time no more than that needed for embedding one sensor. The approach is dipping the actuator array into a graphite solution to coat all the actuators at one time. However, the signal conditioner is costly, regardless of the fact that it is already much cheaper than similar products on market. (\$25 each compared to an LVDT signal conditioner which is $\$ 100 \sim 300$ )

Using careful design, CCRS sensor can be made to respond very rapidly. The output signal stabilizes in less than $0.5 \times 10^{-6}$ seconds after excited, facilitating the usage of a multiplexing scheme to share one signal conditioner among all the sensors. For detailed information please refer to previous publications [12].

\section{Pressure Sensing}

As a human computer interface, methods to detect the user contact are required. Using a pressure sensor array is a conventional but is the easiest approach. A pressure sensor array can be fabricated on one or several wafers. Since building this kind of sensor array is costly and conventional, the original approach focused on a single cell system which needs only one pressure sensor. (Fig 8a) In Fig 8b, the testing result is presented. With the pressure sensor, the system can simulate a spring (curve a-b) and a plastic deformation (curve $b-c$ ). Note that the pressure in the cylinder (vertical axis) is proportional to the user's downward force on the cylinder tip.

Besides making the whole pressure array on wafer, the following approach depicts an alternative by attaching diced pressure sensors onto a less expensive substrate. (Fig 9)
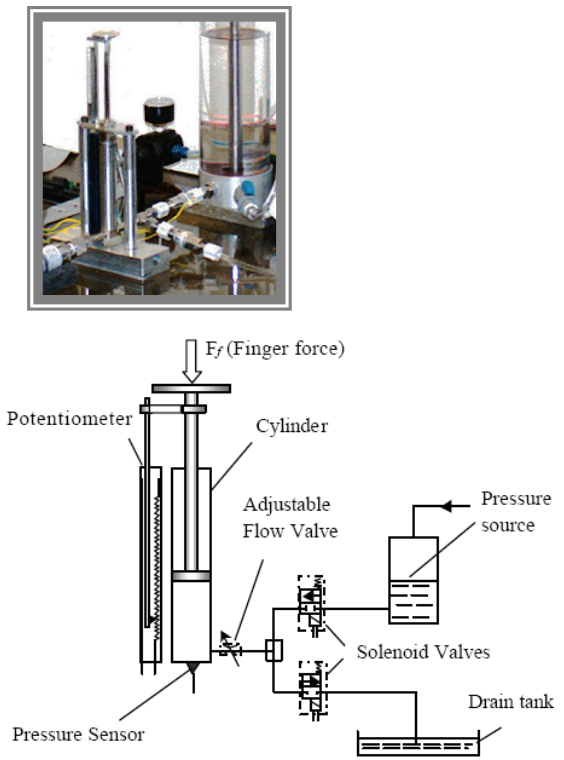

a) Single Cell Testing System

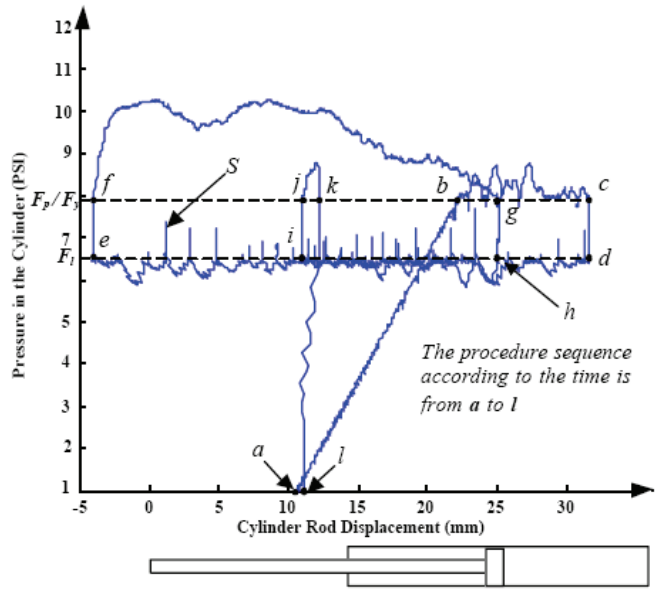

b) Experimental Result

Fig 8 Single Cell Testing

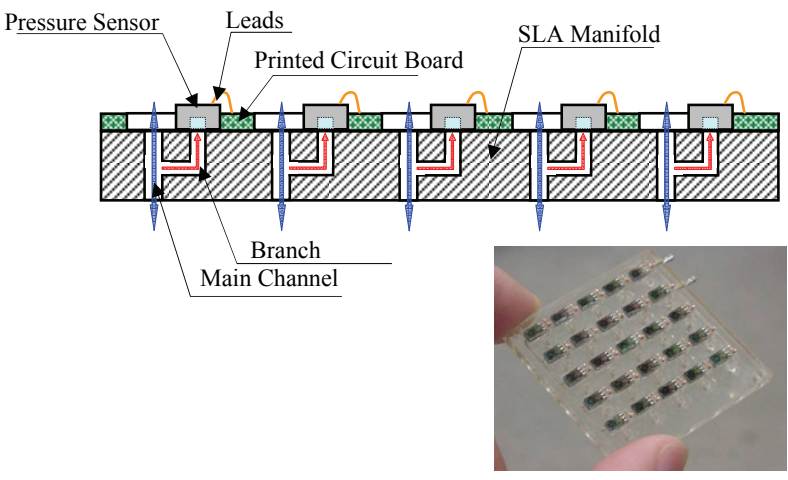

Fig 9 Pressure Sensor Array

\section{HIGH LEVEL CONTROL}

Compared to the fluid driving and actuator-sensor array, the high level control scheme is relatively conventional. It is realized using Real Time Linux. Multi-threading programming enables each module of the control system to be running almost simultaneously. The current control structure for the $5 \times 5$ prototype is presented in Fig 10 . 


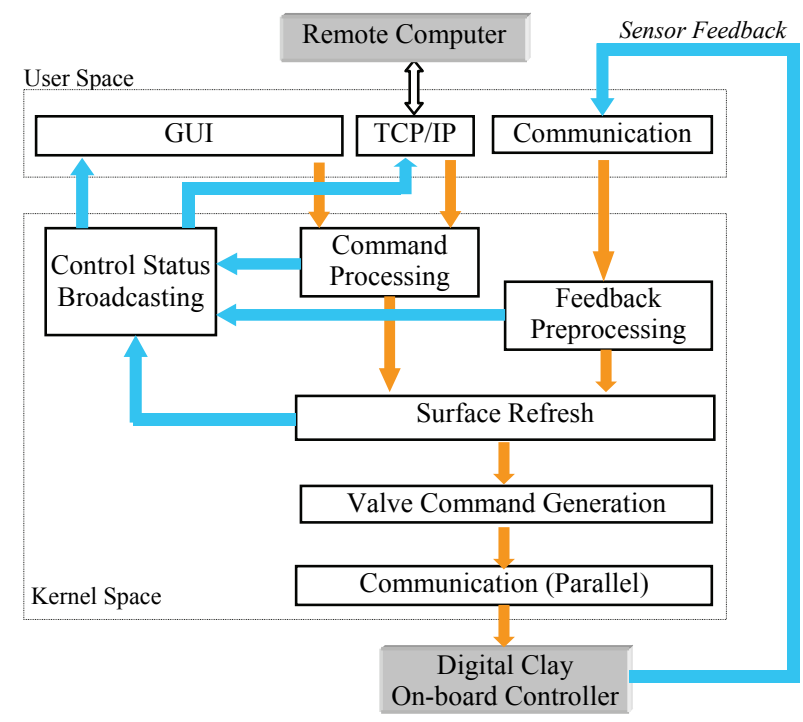

Fig 10 General Control Structure of Digital Clay

Information transferred between modules uses matrices. At the current stage, the surface displacement matrix is the only one used. More information matrices such as material property matrix will be investigated and implemented. Sending information in the format of a matrix makes the message compact and easy to understand.

Surface control is based on the one-time surface refresh method described in section 2 . The surface refresh module compares the desired surface matrix with the current feedback surface matrix, and gives out the reference speed for each actuator in a certain row to the Valve Command Generation module. The Valve Command Generation module calculates the desired PWM duty cycle for each actuator based on the input from the Surface Refresh module.

\section{TEST RESULTS ON A 5X5 PROTOTYPE}

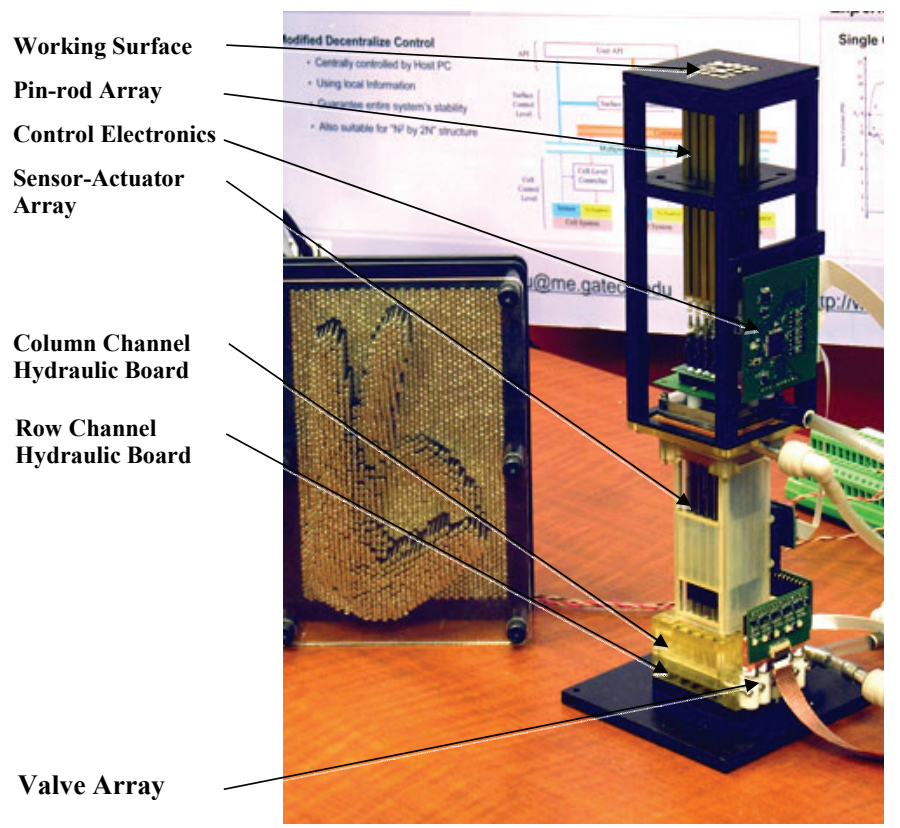

Fig 11 Digital Clay $5 \times 5$ Prototype

At the current stage, a $5 \times 5$ fully functional cell array prototype is designed and implemented as shown in Fig 11.

\section{Basic testing Results for $5 \times 5$ array prototype}

Commanded surface shape:

$\left[\begin{array}{ccccc}16 & 20 & 24 & 28 & 32 \\ 12 & 16 & 20 & 24 & 28 \\ 8 & 12 & 16 & 20 & 24 \\ 4 & 8 & 12 & 16 & 20 \\ 0 & 4 & 8 & 12 & 16\end{array}\right]$

Detected shape by Machine

Vision

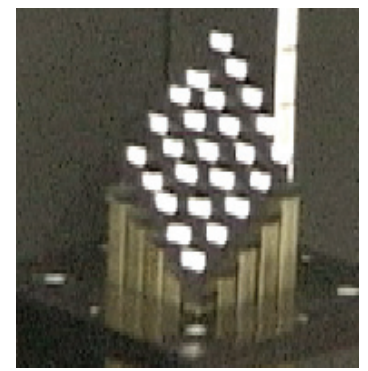

$\left[\begin{array}{ccccc}15.6 & 20.4 & 24.3 & 27.7 & 31.2 \\ 11.7 & 16.5 & 20.8 & 23.8 & 28.7 \\ 7.8 & 12.1 & 16.0 & 20.5 & 24.6 \\ 4.3 & 8.7 & 11.7 & 16.8 & 19.5 \\ 0 & 4.3 & 8.2 & 12.1 & 16.5\end{array}\right]$

Fig 12 Slanted Plane Measured by Camera.

Results included here on the above $5 \times 5$ array prototype are limited due to space but are included to illustrate the satisfactory integrated functioning of all components. The first test is to create a stationary surface. As a test, the working surface is commanded to achieve a slanted plane as shown in Fig 12. The commanded and measured (using machine vision) surface matrices of the working surface given below show less than $0.8 \mathrm{~mm}$ error (no filtering, no calibration). There are many means to measure the final surface. However, machine vision provides a simple, independent way and requires less setup and cost for preliminary experimental purposes.

To test the velocity control ability of the proposed PWM flow control method, a single rod is commanded to track a sinusoidal trajectory. The measured results are shown in Fig 13. The dashed line is the commanded trajectory and the hollow dots represent the measured displacement.

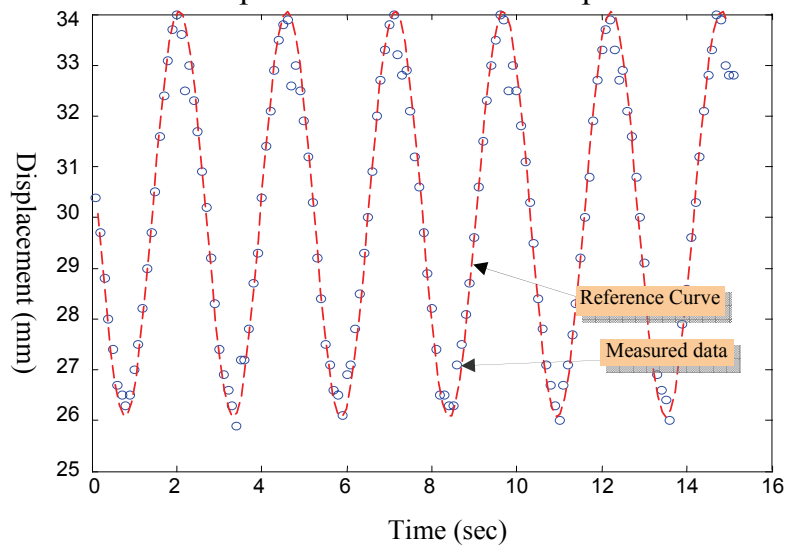

Fig 13 Tracking a Sinusoidal Curve 


\section{FUTURE WORK}

\section{Approximation Gradual Surface Refresh Method Concept}

Both one-time refresh and gradual refresh methods are processed one row after another. To make the surface refresh more efficient, investigation is currently on the possibility of actuating multiple rows simultaneously. This is called approximation gradual refresh method because the intermediate surfaces may be similar but not proportional to the final surface. But the superposition of these intermediate surfaces yields the final surface. Using this approach, the total refresh time can be reduced and a smoothly changing surface may be achieved.

\section{Contact Detection without Pressure Sensor}

As mentioned in the working principle of FMD, section 2, if using air as the fluid media for control coupler and if the user force is large enough, the diaphragm will deflect even if the control coupler is disabled. (Fig 14) Fluid inside the actuator could leak, through the column control channel, into other actuators on the same column. Once this happens, displacement errors will be sensed. As a result, the surface refresh module will try to compensate the error. However, since the rod is pressed by the user, the error will not be compensated. If the situation continues for 0.05 second, (i.e. $20 \mathrm{~Hz}$, which beyond the fastest hand motion the human can perform), this actuator is defined by the control system as being in contact. After all contacted actuators are found (i.e. the whole surface is refreshed), the control system shifts into shaping state.

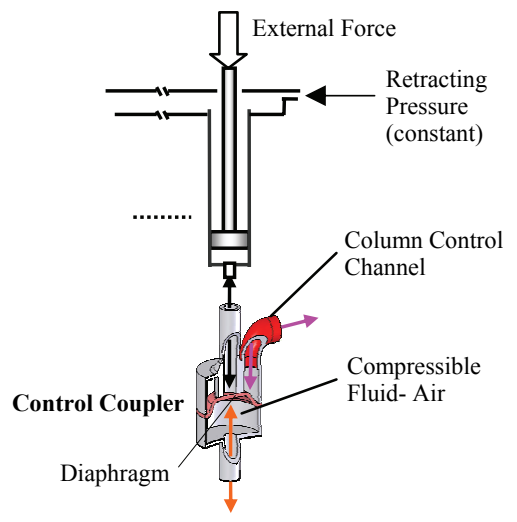

Fig 14 Contact Detection without Pressure Sensor

During the shaping state, these contacted actuators will be linked to the high pressure (keep going upwards). If the user force overcomes the high pressure, the user can push the cylinder rods down slowly. Otherwise, the cylinder rods will go up. Once the desired shape is reached, the user can quickly remove his/her hand. If the speed of a certain actuator is detected to be higher than a threshold, this actuator is will be shifted back to the normal state by the system. Obviously, when shaping the surface, the user needs to keep the motion slow. The actuator will follow the user's hand. Note that, without the pressure sensors, the Digital Clay can be shaped, but it is difficult to simulate material properties like elastic and plastic. But it still provides a good molding device similar to clay.

Other future works are described below. For the prototype development, further work is required to reduce the cost and optimize the structure in order to build an array consisting of more actuators. Currently, each cell is still made separately. A practical solution to make the whole cell array in one pass is under investigation. For sensor signal multiplexing, solutions are under test to allow gathering large amounts of measurements from the cell array. Peripheral devices such as pressure source, control hardware and high level user application interface are still physically large. Compact and efficient devices will be developed to make the device portable.

\section{CONCLUSION}

Digital Clay is a novel 3D computer input/output device. This paper describes a fluid actuated planar array, known as a "Bed of Nails". Included are the issues related to the high level control. A novel actuation circuit suitable for scaling to fine resolution and large areas termed as Fluidic matrix Drive is introduced. The surface control concept is described. These control concepts have been developed in conjunction with the hardware components and their integration.

\section{ACKNOWLEDGMENTS}

This work was supported by the U.S. National Science Foundation ITR grant IIS-0121663. The assistance of the co-investigators (Mark Allen, Ari Glezer, John Goldthwaite, David Rosen, Jarek Rossignac, Imme Ebert-Uphoff, and Chris Shaw) and their colleagues and graduate students in the Schools of Mechanical Engineering and Electrical and Computer Engineering, and the College of Computing is gratefully acknowledged.

\section{REFERENCES}

[1] Fearing, R. S., G. Moy, and E. Tan. Some Basic Issues in Teletaction. In Proceedings of the IEEE Int. Conf. Robotics and Automation, April 1997, pp 3093-3099.

[2] Howe, R. "Tactile sensing and control of robotic manipulation." Advanced Robotics, v. 8 N. 3, 1994, pp245261.

[3] Kammermeier, P. M. Buss, and G. Schmidt, "Dynamic display of distributed tactile shape information by a prototypical array," Proceedings of the 2000 IEEE/RSJ International Conference on Intelligent Robotics and Systems, pp 11191124.

[4] Cohn, M., M. Lam, and R. Fearing, "Tactile feedback for teleoperation," SPIE Telemanipulator Technology, 1833:240$254,1992$.

[5] Howe RD, Peine WJ, Kontarinis DA, Son JS. Remote palpation technology. IEEE Engineering in Medicine and Biology, 14(3):318-323, May/June 1995.

[6] http://www.star.t.u-tokyo.ac.jp/projects/popup, Dec, 2005 
[7] H. Iwata, H. Yano, F. Nakaizumi, and R. Kawamura, "Project FEELEX: Adding haptic surface to graphics," in Proceedings of SIGGRAPH2001, 2001.

[8] G.J. Pappas, G. La®eriere, \& S.S. Sastry, Hierarchically Consistent Control Systems," IEEE Trans. Automatic Control, 45(6) 1144-1160, June, 2000.

[9] Drouin, M., Abou-Kandil, H., and Mariton, M., Control of Complex Systems, Plenum Press, New York, 1991.

[10] D. Gavel and D. Siljak, Decentralized adaptive control: structural conditions for stability, IEEE Trans. Autom. Contr. 34 (1989), no. 4, 413-426.

[11] J. Baillieul, Feedback Designs for Controlling Device Arrays with Communication Channel and width Constraints, in Lecture Notes of the Fourth ARO Workshop on Smart Structures, Penn State Univ., August 16-18, 1999.

[12] H.Zhu, W. Book, "Construction and Control of Massive Hydraulic Micro-actuator-sensor Array", IEEE International Symposium on Computer-Aided Control Systems Design, October, 2006, Munich, Germany

[13] W. Book, H.Zhu, "Haptic Surfaces through Mechatronic Design", IEEE International Conference on Cybernetics and Intelligent Systems (CIS)and Robotics, Automation and Mechatronics (RAM), June,2006, Bangkok, Thailand

[14] H. Zhu, W. Book, "Embedding and Multiplexing Large Scale Sensor Arrays for Digital Clay", Proc. ASME International mechanical Engineering Congress and Exposition, Orlando, 2005.

[24] H. Zhu, "Practical Design and Control for Digital Clay", $\mathrm{PhD}$ Thesis 